\title{
MODELO DE EVALUACIÓN DEL IMPACTO EN ANDALUCÍA DE LAS OBRAS CIVILES REALIZADAS CON MOTIVO DE LA EXPOSICIÓN UNIVERSAL DE SEVILLA 1992
}

\author{
José Javier RODRÍGUEZ ALCAIDE *
}

\section{INTRODUCCIÓN.}

Han pasado ya casi sesenta años desde que el enfoque original de Leontief (1936) de caracter input-output abrió un nuevo camino de análisis y proyección del entramado económico. Desde entonces hasta hoy, de modo creciente, rápido y vigoroso han florecido numerosas áreas de estudio y debate alrededor del análisis input-output. Tantos logros vienen a reafirmar la validez de esta línea de pensamiento que pretende dar respuesta a multiples cuestiones económicas relacionadas con la realidad de los procesos productivos y de intercambio.

$\mathrm{El}$ análisis input-output ha ayudado a los procedimientos de recogida de datos y a la clasificación de la metodología de dicha recogida. También ha ayudado al diseño de modelos económicos que contemplan restricciones en las capacidades de producción y asimismo el comportamiento optimizador de los agentes sociales. El análisis input-output ha iluminado estudios de carácter nacional y regional. Dentro de este ámbito regional hemos trabajado en diferentes ocasiones y de modo especial en esta contribución que ahora aportamos.

\section{DELIMITACIÓN DEL ÁMBITO DEL MODELO Y OBJETIVOS DEL MISMO.}

El modelo no pretende evaluar a medio y largo plazo el impacto de la celebración de la Exposición Universal en Sevilla en 1992 desde la óptica de funcionamiento del acontecimiento.

*. Catedrático de Economía. Universidad de Córdoba. 
El modelo ha pretendido evaluar el impacto en Andalucía que ha supuesto la ejecución de obras civiles necesarias para la celebración del acontecimiento y aceleradas para mejorar la accesibilidad a Sevilla y a la Isla de la Cartuja.

El modelo no ha incorporado, después de un riguroso proceso de previo análisis, las obras que se estaban ejecutando conforme al I Plan de Carreteras o que se habían licitado en 1986 y programado ejecutar desde 1987, previstas con anterioridad al diseño del acontecimiento.

El modelo tampoco ha incorporado para evaluación obras, que se habían incluido por causa de la celebración del acontecimiento y que por causas muy diversas no se han ejecutado total o parcialmente en el periodo 88-92 y que, sin embargo, se habían anunciado en el correspondiente programa.

En resumen, la investigación sólo ha considerado aquellas obras civiles nuevas o aceleradas, programadas por causa de la Exposición Universal y que, definitivamente, se han ejecutado en el periodo temporal del análisis 1988-1992.

\section{EL MODELO DE EVALUACIÓN PARA ANDALUCÍA EN EL PERÍODO 1987-1992.}

\subsection{La estructura deI modelo.}

La estructura del modelo aparece diagramada en la Tabla n- 1. El modelo presenta una forma matricial de 201 columnas y 269 filas. Aparecen en dicha tabla los bloques de columnas X, CT, CAP, ENS, SAN, MPP, FBC y RHS, con la numeración del número de columnas que indican las "actividades" o variables del sistema. Asimismo aparecen los bloques de filas con su numeración representando las relaciones estructurales de las variables. La última columna viene denominada como ECUAClÓN(n), con lo que se quiere indicar la expresión matemática que refleja la relación entre variables y que aparece en el apartado "generación de relaciones estructurales".

El primer bloque $\mathrm{X}$, matriz de orden $(64 \times 64)$ refleja las relaciones estructurales del sistema productivo de Leontieff, en tanto que el bloque (12) de ecuaciones representa las relaciones económicas totales entre producción, consumo, formación bruta de capital y exportaciones al exterior de la región.

EI vector columna RHS señala los parámetros del modelo que harán jugar el sistema económico en función del nivel de exportaciones, consumo privado y público y nivel de formación bruta de capital; asimismo dentro de este vector aparece el subvector $\mathrm{Q}$ que delimita la capacidad de producción del sistema productivo regional. 


\subsection{Generación de las relaciones estructurales del modelo.}

Definicion de las actividades.

$\mathrm{X}_{\mathrm{j}}=$ Es la producción efectiva en términos input-output de la rama j-esima de la economía andaluza valorada cada unidad en $10^{6}$ PTAS.

$C_{j}=$ Es el valor del consumo de hogares suministrado desde Andalucía por la rama i-esima, expresada cada unidad en $10^{6}$ PTAS.

$\mathrm{CAP}_{\mathrm{j}}=$ Es el valor del consumo de las Administraciones Públicas suministrado desde Andalucía por la rama i-esima, expresado en $10^{6}$ PTAS.

$\mathrm{FBC}_{\mathrm{j}}=$ Es el valor de la Formación Bruta de Capital vendida en Andalucía y desde Andalucía para y por la rama i-esima, expresada en $10^{6}$ PTAS.

$\mathrm{EX}_{\mathrm{j}}=$ Es el valor de las exportaciones andaluzas de la rama i-esima al resto de España y al Extranjero.

Definicion de relaciones estructurales

$$
\mathrm{X}_{\mathrm{j}}=\sum \mathrm{a}_{\mathrm{ij}}{ }^{\mathrm{r}} \cdot \mathrm{X}_{\mathrm{j}}+\widehat{\mathrm{C}}_{\mathrm{i}, \mathrm{r}}+\widehat{\widehat{\mathrm{CAP}}} \mathrm{\widehat {i } , \mathrm { r }}+\widehat{\mathrm{FBC}_{\mathrm{i}, \mathrm{r}}}+\mathrm{EX}_{\mathrm{i}}
$$

donde se expresa que:

$X_{j}$ es un vector $(n * 1)$ que representan la producción andaluza de cada rama i-esima.

$\sum \mathrm{a}_{\mathrm{ij}}{ }^{\mathrm{r}} \cdot \mathrm{X}_{\mathrm{j}}$ es una matriz (n*) que representa la parte de la producción de la rama $\mathrm{i}$ que se vende como input intermedio de la rama $\mathrm{j}$. El coeficiente $a_{\mathrm{ji}, \mathrm{r}}$ es el coeficiente regional de Leontief en terminología input-output.

$\hat{\mathrm{C}}_{\mathrm{i}, \mathrm{r}}$

es una matriz diagonal $\left(n^{*} n\right)$, en cuya diagonal aparecerán los coeficientes $C_{i}$, para $i=j$, que representan la proporción que del consumo final de los hogares del sector i es satisfecho por la producción regional del sector $\mathrm{i}$.

$\widehat{C A P}_{i, r}$ es una matriz diagonal $(n * n)$ en cuya diagonal aparece el coeficiente $\mathrm{CAP}_{\mathrm{i}}$ para $\mathrm{i}=\mathrm{j}$, que representa la proporción que del consumo final público de sector i es satisfecha por la producción regional de dicho sector i. 
$\widehat{\mathrm{FBC}}_{\mathrm{i}, \mathrm{r}}$ es una matriz diagonal $(\mathrm{n} * \mathrm{n})$ en cuya diagonal aparece el coeficiente $f_{\mathrm{i}}$, para $\mathrm{i}=\mathrm{j}$, que representa la proporción de formación bruta de capital procedente de la rama i que es satisfecha por la misma rama i desde la región.

$\operatorname{EX}_{\mathrm{i}}$ es un vector $\left(\mathrm{n}^{*} 1\right)$ que representa la cantidad de exportaciones al resto de España y al Extranjero que se realizan desde la región por cada rama $i$.

Los coeficientes $\mathrm{A}_{\mathrm{ij}, \mathrm{r}}$, para el periodo 1988-1991 se toman de la TIOA 87.

Los coeficientes $\mathrm{C}_{\mathrm{i}}, \mathrm{CAP}_{\mathrm{i}}$, también se toman de la TIOA-87 y representan $\mathrm{C}_{\mathrm{i}}=\mathrm{C}_{\mathrm{i}, \mathrm{r}} / \mathrm{C}_{\mathrm{i}} ; \mathrm{CAP}_{\mathrm{i}}=\mathrm{CAP}_{\mathrm{i}, \mathrm{r}} / \mathrm{CAP}_{\mathrm{i}} ; f_{\mathrm{i}}=\mathrm{FBC}_{\mathrm{i}, \mathrm{r}} / \mathrm{FBC}_{\mathrm{i}}$ y se usan para el periodo 1989-1991.

Esta parte del modelo opera transformando [11] en [12]

$$
\left[\mathrm{X}_{\mathrm{j}}-\sum \mathrm{a}_{\mathrm{ij}}^{\mathrm{r}} \cdot \mathrm{X}_{\mathrm{j}}\right]-\widehat{\mathrm{C}}_{\mathrm{i}, \mathrm{r}}-\widehat{\mathrm{CAP}}_{\mathrm{i}, \mathrm{r}}-\widehat{\mathrm{FBC}_{\mathrm{i}, \mathrm{r}}}=\mathrm{EX}_{\mathrm{i}}
$$

de modo que para los conocedores del análisis input-output recordamos que $\left[\mathrm{X}_{\mathrm{j}}-\sum \mathrm{a}_{\mathrm{ij}}{ }^{\mathrm{r}} \mathrm{X}_{\mathrm{j}}\right]$ es la matriz de multiplicadores regionales de Leontief.

El modelo no puede formalizarse sin acotar por arriba la capacidad de producción de cada sector, pues en una simulación de un escenario a cuatro años debe pensarse que la capacidad de producción de cada rama i está limitada a la que actualmente posee en la actualidad. La capacidad de producción de cada rama i depende de su coeficiente actual de utilización efectiva y de su flexibilidad para aumentar turnos de producción. Esta relación de capacidad se representa por la relación (29), como se verá más adelante.

Definición de las relaciones de los componentes de la demanda final y su distribución por rama y por origen.

El modelo ha endogenizado (ver [12]) las variables de consumo en hogares, en AAPP y Formación Bruta de Capital. Acotados límites para estas tres magnitudes globales las relaciones de distribución son las siguientes:

$\mathrm{C}=\mathrm{K}$ donde $\mathrm{C}$ es el consumo total de Andalucía y $\mathrm{K}^{\prime} \leq \mathrm{K} \leq \mathrm{K}^{\prime \prime}$ $\mathrm{C}=\sum \mathrm{c}_{\mathrm{i}}{ }^{*} \mathrm{C}$ donde $\mathrm{c}_{\mathrm{i}}$ es la proporción de la rama $\mathrm{i}$ en el consumo total $\mathrm{C}$. 
[15]

$I=c_{i}$ donde I es la matriz unidad y $c_{i}$ es un vector $(n * 1)$ de coeficientes de distribución por ramas i del consumc total.

[16]

$\mathrm{CAP}=\mathrm{L}$ donde $\mathrm{CAP}$ es el consumo público total de Andalucía y $\mathrm{L}^{\prime} \leq \mathrm{L} \leq \mathrm{L}^{\prime \prime}$.

[17] $\mathrm{CAP}=\sum \mathrm{cap}_{\mathrm{i}} \mathrm{CAP}$ donde $\mathrm{cap}_{\mathrm{i}}$ es la proporción de la rama i en CAP.

[18] I= cap donde I ya es definida y $\operatorname{cap}_{i}$ es un vector $\left(\mathrm{n}^{*} 1\right)$ de coeficientes de distribución por ramas i de CAP.

[19]

$\mathrm{FBC}=\mathrm{M}$ donde $\mathrm{FBC}$ es la Formación Bruta de Capital Total y $\mathrm{M}^{\prime} \leq \mathrm{M} \leq \mathrm{M}^{\prime \prime}$.

[20] $\quad \mathrm{FBC}=\sum f_{\mathrm{i}} \mathrm{FBC}$ donde $f_{\mathrm{i}}$ es la proporción de la rama i en la $\mathrm{FBC}$ total.

$\mathrm{I}=\mathrm{f}$ donde $\mathrm{I}$ es conocida y $f_{\mathrm{i}}$ es un vector $(\mathrm{n} * 1)$ de coeficientes de distribución por ramas i de la FBC.

El vector Cj varía para el periodo 1988-91 en tanto que se mantienen con la misma estructura los vectores cap y $f_{\mathrm{i}}$.

Definición de la balanza comercial del sistema productivo.

Para el análisis del modelo "Andalucía con EXPO" conviene incorporar las relaciones comerciales del sistema andaluz con el exterior. Así

$\mathrm{B}=$ EXPORTACIONES MENOS IMPORTACIONES DE [BIENES INTERMEDIOS + BIENES DE CONSUMO + BIENES DE INVERSIÓN]

Esta expresión se ha modelizado según [22]

$$
\begin{aligned}
& \text { B }=\sum \mathrm{e}_{\mathrm{i}} \cdot \mathrm{x}_{\mathrm{j}}-\sum \mathrm{m}_{\mathrm{j}} \cdot \mathrm{x}_{\mathrm{j}}-\sum \mathrm{n}_{\mathrm{i}} \cdot \mathrm{c}_{\mathrm{i}}-\sum \mathrm{q}_{\mathrm{i}} \mathrm{FBC}_{\mathrm{i}}, \quad \text { que reordenada } \\
& \sum\left(\mathrm{e}_{\mathrm{j}}-\mathrm{m}_{\mathrm{j}}\right) \cdot \mathrm{X}_{\mathrm{j}}-\sum \mathrm{n}_{\mathrm{i}} \cdot \mathrm{c}_{\mathrm{i}}-\sum \mathrm{q}_{\mathrm{i}} \cdot \mathrm{FBC}_{\mathrm{i}} \stackrel{\leq}{\leq}
\end{aligned}
$$


donde:

$e_{j}$, es el porcentaje de la producción efectiva $X_{j}$ que va al exterior de Andalucía.

$\mathrm{m}_{\mathrm{j}}$ es el porcentaje de $\mathrm{X}_{\mathrm{j}}$ que constituyen inputs intermedios importados fuera de Andalucía.

$n_{i}$ es el porcentaje que de cada unidad de consumo $C_{i}$ viene de exterior a la región y es el complementario de $\mathrm{C}_{\mathrm{i}, \mathrm{r}}$.

$\mathrm{q}_{\mathrm{i}}$ es el porcentale que de cada unidad de $\mathrm{FBC}_{\mathrm{i}}$ viene exterior a la región y es el complementario a $\mathrm{FBC}_{\mathrm{i}, \mathrm{r}}$.

Los coeficientes $e_{j}, m_{j}, n_{i}, q_{i}$, se obtienen de la TIOA-87 y se mantienen constantes durante 1988-1989.

Definición de indicadores económicos.

Para el modelo de referencia interesa añadir las siguientes relaciones.

[24] $I=\sum m_{j} \cdot X_{j}+\sum q_{i} \cdot F B C i, \quad$ donde I son importaciones intermedias y de bien de inversión.

[25] $\mathrm{VAB}=\sum \mathrm{v}_{\mathrm{i}} \cdot \mathrm{X}_{\mathrm{j}}$ donde $\mathrm{v}_{\mathrm{j}}$ es el coeficiente de valor añadido bruto unitario de la rama $\mathrm{j}$.

[26] $T=\sum t_{j} \cdot X_{j} \quad$ donde $t_{j}$ es el coeficiente unitario de empleo de la rama $j$.

[27] $t_{j, t+1}=t_{j, t} \cdot\left(1+p_{i}\right)$ donde $p$ es la tasa de aumento de la productividad del trabajo en la rama $j$, siendo $t=1987$.

Definición de la función objetivo:

[28] Minimizar las desviaciones positivas al vector $\mathrm{EX}_{\mathrm{i}}$ sujeto a:

[12], [13], [15], [16], [18], [19], [21], [23], [24], [25], [26] y [29].

[29] $Q_{j}{ }^{t} \geq X_{j}{ }^{t}$ donde $Q_{j}$ es la capacidad productiva del sector $j$ en $t=1987$. 


\subsection{La lógica del modelo.}

De acuerdo con lo expuesto en los puntos 2.1 y 2.2, el modelo pretende simular la evolución del sistema económico andaluz del siguiente modo:

Minimizando las desviaciones positivas al vector de ventas al exterior $\mathrm{EX}_{\mathrm{i}}$, de modo que se entiende que es el mercado externo el que origina el crecimiento de Andalucía, siempre que se cumplan las siguientes condiciones:

a) que se de equilibrio entre la producción de origen andaluz y las ventas intermedias y finales (consumo privado, público, formación de capital y ventas al exterior de la región).

b) que el consumo total regional se mueva alrededor de una cifra $\mathrm{K}$ precalculada, de acuerdo con las hipótesis extemas del modelo (seguimiento de la traza de crecimiento nacional).

c) que este consumo global se descompone siguiendo una determinada pauta, también determinada externamente al modelo y que esta pauta sectorial condiciona el mercado de proveedores regionales de acuerdo con la matriz $\mathrm{C}_{\mathrm{ir}} / \mathrm{C}_{\mathrm{i}}$

d) que el consumo público se acota entre valores L' y L" y se distribuye regionalmente en 5 sectores entre lo más importante el de enseñanza, sanidad y administraciones públicas.

e) que la formación bruta de capital se acota entre valores exogenamente predeterminados $\mathrm{M}$ y se sectoriza de acuerdo con la pauta del vector (136) denominado $\mathrm{FBC}_{\mathrm{i}} / \mathrm{FBT}$.

f) que la balanza comercial de la región cumple las condiciones explicitadas en las ecuaciones (23) y (24), a fin de conocer su saldo de evolución.

g) que las ecuaiones (25) de valor añadido y (26) de empleo nos permitan conocer el valor global regional producido por el sistema.

h) que de acuerdo con (29) se respete que la capacidad productiva del sistema a nivel sectorial no puede ser sobrepasada, de modo que la región importaría los bienes y servicios necesarios. 


\section{REQUERIMIENTOS DE INFORMACIÓN.}

1 - Matriz $A_{R}[64 \times 64]$

Tomar matriz de coeficientes regionales de LEONTIEF y restar de matriz I

2 - Matriz $\hat{C}_{i}[64 \times 64]$

Tomar el vector de la demanda final denominado "Consumo de hogares".

Dividir la cifra de ventas regionales por el consumo total de cada rama.

3 - Matriz $\widehat{\mathrm{FBC}}$ [ $[64$ x 64]

Tomar el vector de la demanda final denominado "formación bruta de capital". Dividir la cifra de origen regional por la cifra total en cada rama.

4 - Vector CT $(64 \times 1)$

Tomar el vector denominado "Consumo de Hogares". Dividir el Consumo total de cada rama por el consumo total regional.

5 - Vector CAP $(5 \times 1)$

Tomar el vector denominado "consumo público". Dividir el consumo de cada rama por el consumo público total.

6 -Vector FBC $(64 \times 1)$

Tomar el vector denominado "formación bruta de capital". Dividir la formación de capital de cada rama por la formación capital regional.

7 - Vector $\mathrm{C}(1 \times 64)$

Tomar el vector de exportaciones totales y tomar el vector "producción efectiva". Dividir cada elemento del vector "exportaciones" por el correspondiente del vector "producción efectiva".

8 - Vector m (1 x 64)

Tomar el vector de importaciones intermedias totales y dividirlo por el vector de producción efectiva.

9 - Vector n $(1 \times 64) \wedge$

Tomar la matriz $\mathrm{C}_{\mathrm{i}}$ y restarla de $\mathrm{I}$.

10 -Vector q $(1 \times 64)$

Tomar la matriz $\mathrm{FBC}_{\mathrm{i}}$ y restarla de I. 
11 - Vector v $(1 \times 64)$

Tomar vector valor añadido y dividirlo por vector "producción efectiva"

12 - Vector $\mathrm{t}(1 \times 64)$

13 - Vector EX $(64 \times 1)$

Tomar vector "exportaciones" de 1987

14 - Vector EX (64 x 1) del año T+1

- Tomar vector EX del año T

- Tomar tasa de crecimiento del consumo y de la inversión de la ECONOMÍA NACIONAL o bien de la renta.

- Aplicar esta tasa a las ramas correspondientes.

- Multiplicar este vector por el vector elasticidad renta para el consumo de las ramas distintas a las de inversión, si se usa la renta.

a) $\mathrm{EX}(\mathrm{T}+1)=\mathrm{EX}(\mathrm{T}) * \delta \mathrm{Y}$ esperado $* \varepsilon$

b) $\mathrm{EX} 1(\mathrm{~T}+1)=\mathrm{EX}_{1}(\mathrm{~T}) * \delta$ consumo

EX1 $(\mathrm{T}+1)=\operatorname{EX} 1(\mathrm{~T})+\delta$ inversión.

15 - Obtención de $\mathrm{K}(\mathrm{T}+1)$

$\mathrm{K}(\mathrm{T}+1)=\mathrm{K}(\mathrm{T})+\delta$ Población $* \mathrm{~K}(\mathrm{~T})+\varepsilon \delta \mathrm{Y} * \mathrm{~K}(\mathrm{~T})$.

$\varepsilon$, un vector de $(64 \times 1)$ de elacticidades-renta por ramas de consumo.

16 - Obtención de $\varepsilon$

Tomar encuestas presupuestos familiares $1971-73$ y $1981-83$ por productos para A.

Tomar renta familiar disponible 1971-73 y 1981-83 para Andalucía.

Expresar gastos y renta en pesetas constantes de 1981-83.

Obtener: Elasticidad.

$$
\epsilon_{i}=\frac{\frac{\text { Gasto } 83-\text { Gasto } 73}{\text { Gasto } 73}}{\frac{\text { Renta } 83-\text { Renta } 73}{\text { Renta } 73}}
$$


17 - Obtención de CT $(\mathrm{T}+1)$

$$
C T(T+1)=\frac{C 1+\delta P * C 1(T)+\epsilon 1 \delta Y C 1(T)}{K(T+1)}+\ldots+\frac{C 64+\delta P * C 64(T)+\epsilon 64 \delta Y C 64(T)}{K(T+1)}
$$

18 - Obtención de $\mathrm{L}(\mathrm{T}+1)$

$$
\mathrm{L}(\mathrm{T}+1)=\mathrm{L}(\mathrm{T})+\delta \text { CONSUMO PÚBLICO }
$$

19 - Obtención de M (T + 1)

$$
\mathrm{M}(\mathrm{T}+1)=\mathrm{M}(\mathrm{T}) \delta \text { INVERSION }
$$

20 - Obtención del vector $\mathrm{T}(\mathrm{T}+1)$

$\mathrm{t}(\mathrm{t}+1)=\mathrm{t}(\mathrm{T}) *$ vector de Productividades.

\section{RESULTADOS.}

Los resultados de la simulación del modelo han sido publicados recientemente en el Boletín Económico de Andalucía '. En dicha publicación aparecen todos los inputs del modelo así como los resultados de los escenarios simulados a lo largo del periodo 1989-1992, con la evolución de las magnitudes más relevantes, desde la evolución de la formación bruta de capital, empleos totales brutos generados, consumo privado y efectos sobre los sectores suministradores de insumos.

\section{BIBLIOGRAFÍA.}

BRODY, A y CARTER, A.P. (edtores). (1972): Input-Output Techniques. North Holanc Pub. Co. Amsterdam.

DORFMAN, R; SAMUEISON P. y SOLOW R.W. (1958): Linnear ?rogramming and iconomic Analysis. Mc. Graw-Hill. New York.

ISARD. W. (1951): Intemational and regional I-O analysis: a model of space economy. Rev. of Economic and statistics 33(4)-318-28.

ISSAEV, B; NIJKAMP, P y otros (editores) (1982): Multiregional Economic modelli..1g. North Holland Pub. Co Amsterdam.

POLANSKE, K.R. (1980): The U.S. Multiregional I-O Accounts and Model. Heath and Co. Lexington. Mass.

RODRÍiGUEZ ALCAIDE, J.J. (1992): Evaluación de las obras civiles realizadas en Andalucia con motivo de la Exposición Universal de Sevilla 1992. Boletín Económico de Andalucía (15i): 13-21.

1. Rodríguez Alcaíde J.J. 1992 Boletín Económico Andalucía (15): 13-21. 
TABLA NQ 1

EstRucture DEL MOOELO

cos 200

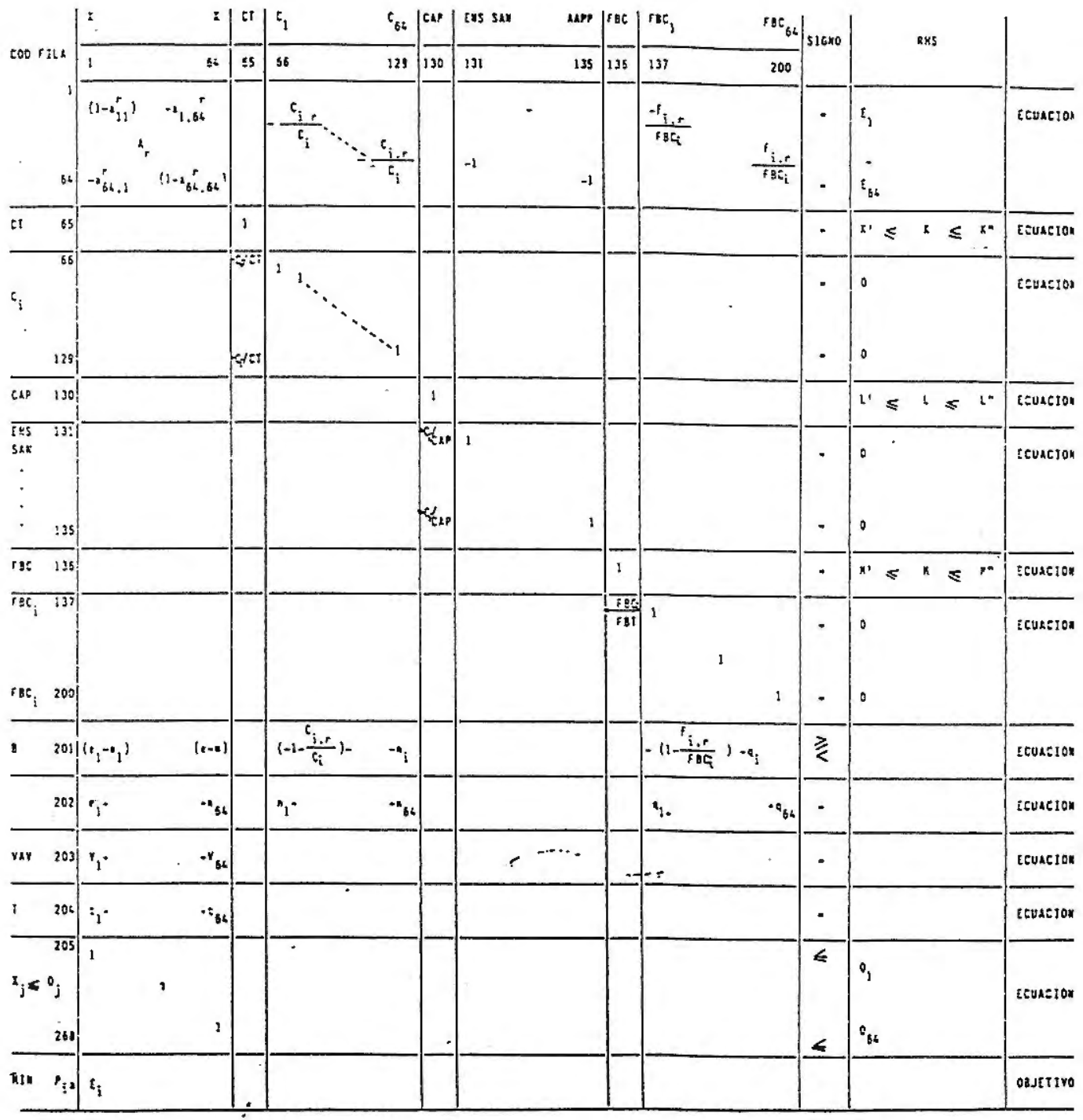

